\title{
Decoupled rotational motion control for visual walking stabilization
}

\author{
Yosuke Asano \\ Kisarazu National College of Technology \\ 2-11-1, kisarazushi, chiba \\ Email: asano@e.kisarazu.ac.jp
}

\author{
Atsuo Kawamura \\ Yokohama National University \\ 79-5, yokohamashi, kanagawa \\ Email: kawamura@ynu.ac.jp
}

\begin{abstract}
A definition of the visual walking proposed by authors is that the robot autonomously walks by making decision based on the image feature motion. One of the achievements is "visual tracking walk". In the past conference, authors proposed a hybrid control for "visual walking". In this paper, the four vertexes of a square surrounding the red target in the image plane are selected as image features. Using such image features, the robot moves in front of the desired position. The rotation orientation of the robot is improved by the feature values caused by the walking motion. The proposed control law is verified by simulations and experiments.
\end{abstract}

\section{INTRODUCTION}

There is almost no report covering the biped walk tracking based on the camera image mounted on the walking robot, and the visual servoing by the posture change for the purpose of the target image tracking in the camera frame. The difficulties can be explained for the three main reasons; (1) since the biped walking is a kind of a cyclic movement without the permanent contact to the ground, the camera image has the periodical disturbance within one walking cycle. This disturbance is very large, and other types of robots such as a wheel driven robot or the standing robot on the ground have no disturbance like this. (2) The biped walking planning is prepared before the actual walking, thus the change of the walking conditions such as the slide width, directions, and the posture has essentially the large time delay in the control loop. (3) An individual robot has physical restrictions due to the hardware structure. For example, the biped walking robot MARI-2[1] cannot bend the body forward, left or right for tracking the target.

Authors have proposed the visual walking, in which the robot autonomously observes feature value and modifies walking trajectory reference in the walking cycle[2]. The visual tracking walk is different from the visual tracking [3], in which the wheel based robot has the camera and the control period is much shorter than that of the visual biped tracking walk. In this paper, authors first consider the stabilization conditions of visual walking when the feature value amplitude and the four vertexes of a square surrounding the red target in the image plane are selected as the new feature value. Second, the simulation and experimental results are reported. The basic algorithm for the visual servo is based on the imagebased visual servo, in which the controlled values are the image features of the object (this scheme is also called feature based)[4][5]. The hardware for the biped walking robot is MARI-2, which are developed by author's group[1].

\section{SySTEM OF THE VisuAL WALKING}

The visual walking system configuration is shown in Fig. 1, which is composed of the environment recognition around the biped robot including the feature extraction, decision making and generation of the walking trajectory.

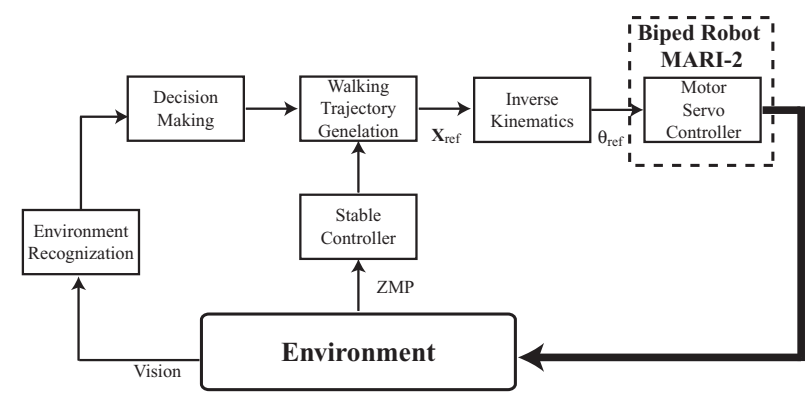

Fig. 1. Visual Walking System Configuration

\section{A. Environment Recognition}

In [2], the median and area of target in the image plane were selected as the feature value. Thus, only translation motion was possible. Here, to extract the rotation motion of the target, first the largest target in the image plane are extracted, and second vertexes of the square which surround this target in the image plane are calculated, and third, position information of the vertexes is used as feature value.

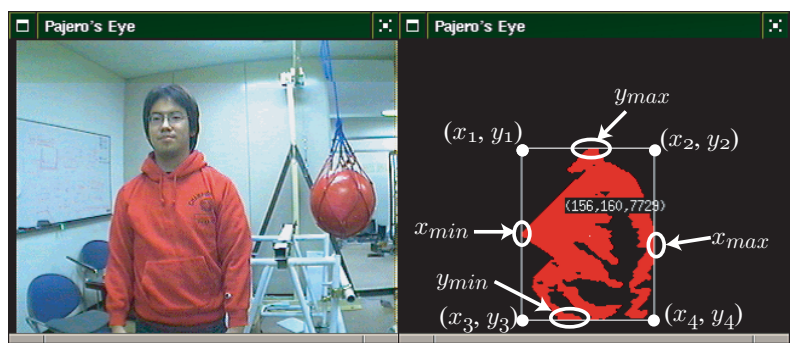

Fig. 2. Environment Recognition (most biggest red target extraction) 


\section{B. Formulation of General Visual Servo System}

The CCD camera is mounted on the head as shown in Fig. 3 , and the coordinates for the camera and the body of the robot are defined as $\{C\}$ and $\{B\}$. The red target, which has 6 -DOF, is set in front of the robot. And image features are selected to be derivative of the vertex position of a square as shown in Fig. 3.

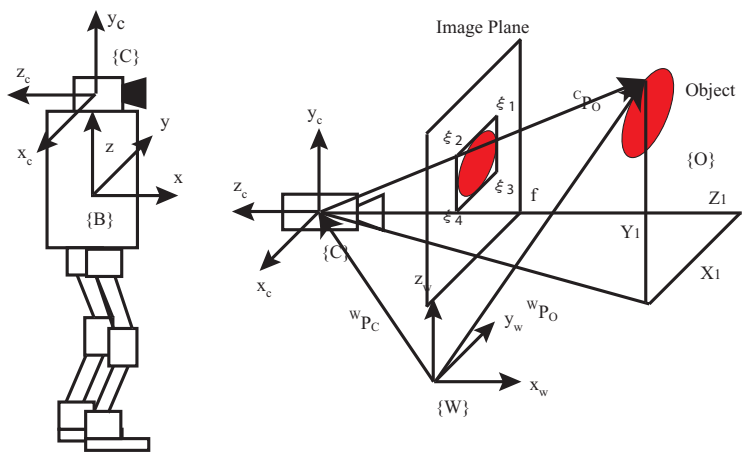

Fig. 3. Body and Camera of Biped Walkin Robot MARI-2 Coordinate System

The CCD camera normally has 6-DOF in the world coordinate system. However, DOF is limited to stabilize the walking. So, The CCD camera has 3-DOF, which is the translation of $\mathrm{Y}$-axis and $\mathrm{X}$-axis and rotation of $\mathrm{Z}$-axis with respect to the body coordinate system. The velocity vector of the camera $\left(\dot{\boldsymbol{x}}_{c}\right)$ and the velocity vector of the image features $(\dot{\boldsymbol{\xi}})$ have the following relation.

$$
\dot{\boldsymbol{\xi}}=\boldsymbol{J}_{3} \dot{\boldsymbol{x}}_{c}
$$

, where $\boldsymbol{J}_{3} \in R^{8 \times 3}$ is called the image Jacobian in [5]. $\dot{\boldsymbol{x}}_{c}=$ $\left[\boldsymbol{v}_{c}, \boldsymbol{\omega}_{c}\right]^{T}$ is the camera translation velocity $\boldsymbol{v}_{c}=\left[v_{x}, v_{z}\right]^{T} \in$ $R^{2}$ and rotation velocity $\boldsymbol{\omega}_{c}=\left[\omega_{z}\right]^{T} \in R^{1}$ with respect to the camera coordinate system $\{\mathrm{C}\}$, and $\dot{\boldsymbol{\xi}}=\left[\dot{\boldsymbol{\xi}}_{1}, \dot{\boldsymbol{\xi}}_{2}, \dot{\boldsymbol{\xi}}_{3}, \dot{\boldsymbol{\xi}}_{4}\right]^{T}=$ $\left[\dot{x}_{1}, \dot{y}_{1}, \dot{x}_{2}, \dot{y}_{2}, \dot{x}_{3}, \dot{y}_{3}, \dot{x}_{4}, \dot{y}_{4}\right]^{T} \in R^{8}$ is features velocity.

The walking planning is made at each step, thus the linearization near the desired features $\boldsymbol{\xi}_{d}$ and the multiplying the walking period $\Delta T$ to (1) yields the following simplified sampled data model.

$$
\overline{\boldsymbol{\xi}}(k+1)=\overline{\boldsymbol{\xi}}+\boldsymbol{J}_{3 d} \boldsymbol{u}_{3}(k) \Delta T
$$

, where $\overline{\boldsymbol{\xi}}=\boldsymbol{\xi}-\boldsymbol{\xi}_{d}, \boldsymbol{J}_{3 d} \in R^{8 \times 3}$ is image Jacobian in desired features, which is given as $\mathrm{Eq}(3)$.

$$
\boldsymbol{J}_{3 d}=\left[\begin{array}{ccc}
-\frac{f}{Z} & \frac{x_{1}}{Z} & -f-\frac{x_{1}^{2}}{f} \\
0 & \frac{y_{1}}{Z} & -\frac{x_{1} y_{1}}{f} \\
-\frac{f}{Z} & \frac{x_{2}}{Z} & -f-\frac{x_{2}^{2}}{f} \\
0 & \frac{y_{2}}{Z} & -\frac{x_{2} y_{2}}{f} \\
-\frac{f}{Z} & \frac{x_{3}}{Z} & -f-\frac{x_{3}^{2}}{f} \\
0 & \frac{y_{3}}{Z} & -\frac{x_{3} y_{3}}{f} \\
-\frac{f}{Z} & \frac{x_{4}}{Z} & -f-\frac{x_{4}^{2}}{f} \\
0 & \frac{y_{4}}{Z} & -\frac{x_{4} y_{4}}{f}
\end{array}\right]
$$

$\boldsymbol{u}_{3}(k)$ is control input $\boldsymbol{u}_{3}(k)=\left[\begin{array}{lll}v_{x}, & v_{z}, & \omega_{z}\end{array}\right]^{T}=$ $\left[u_{x}(k), u_{z}(k), u_{r}(k)\right]^{T}$.

\section{Proposal of the rotation target tracking system}

the previous control law (Generalized Inverses Matrix law) with the feature value selection has not been achieved the rotation control[2]. In [6], Peter et al. proposed hybrid method that decouple the $\mathrm{Z}$-axis motion using new feature value to solve the camera retreat problem. We achieve the aim with the characteristic of the walking. The camera mounted robot sways from side to side in the walking cycle. The sway of the camera causes the oscillation of the feature point in the image plane. The model of the target rotation and the sway of the camera causes oscillation is shown in Fig.4 and the feature value amplitude $\left(\alpha_{1}, \alpha_{2}\right)$ is shown in Fig.5, where $L$ is the width of the target, $A_{c}$ is the camera amplitude by the walking, $Z$ is distance between the camera and target object. $\theta$ and $\theta_{c}$ are Z-axis rotation angle of the target and camera with respect to the world coordinate system.

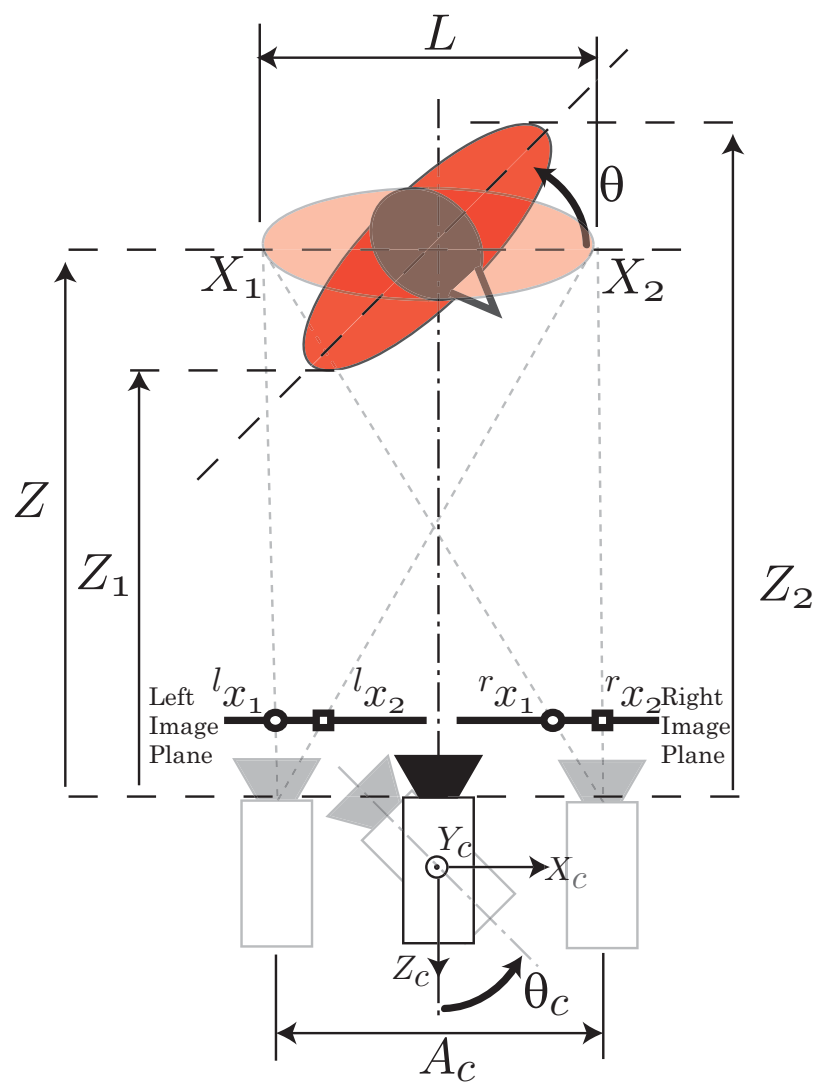

Fig. 4. Model of target rotation and oscillation of feature value in the image plane

The camera captures the images at the both ends of the oscillation by the walking. (Fig.5). The feature value difference $\alpha_{1 d}$ is difference between the feature value ${ }^{r} x_{1}$ in the right 


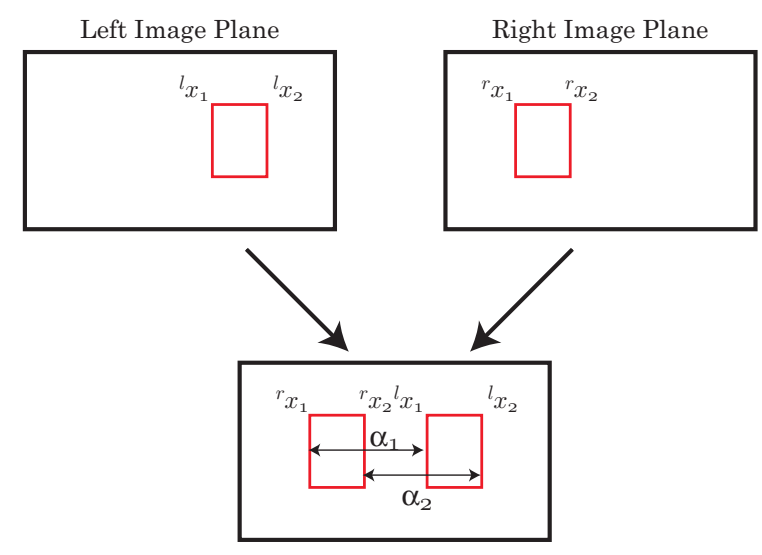

Fig. 5. Ocillation of feature value $\alpha_{1}, \alpha_{2}\left(\alpha_{1 d}, \alpha_{2 d}\right)$ by camera motion

image plane and ${ }^{l} x_{1}$ in the left image.

$$
\begin{aligned}
\alpha_{1 d} & ={ }^{r} x_{1}-{ }^{l} x_{1} \\
& =\left(\frac{A_{c}}{2}-X_{1}\right) \frac{f}{Z}-\left(-\frac{A_{c}}{2}-X_{1}\right) \frac{f}{Z} \\
& =A_{c} \frac{f}{Z}
\end{aligned}
$$

$\alpha_{2 d}$ also has the following relation.

$$
\alpha_{2 d}={ }^{r} x_{2}-{ }^{l} x_{2}=A_{c} \frac{f}{Z}
$$

The feature value difference $\alpha_{1}$ and $\alpha_{2}$ have the following relation when the target object rotates $\theta$ around the Z-axis with respect to the world coordinate system.

$$
\begin{aligned}
& \alpha_{1}=A_{c} \frac{f}{Z_{1}}=A_{c} \frac{f}{Z-\frac{L}{2} \sin \theta} \\
& \alpha_{2}=A_{c} \frac{f}{Z_{2}}=A_{c} \frac{f}{Z+\frac{L}{2} \sin \theta}
\end{aligned}
$$

Eq.(6) and (7) is nonlinear equation, so that they yield following equation by linearization near $\theta=0$.

$$
\begin{aligned}
\alpha_{1} & \sim A_{c} \frac{f}{Z}+A_{c} \frac{f}{2 Z^{2}} L \theta \\
\alpha_{2} & \sim A_{c} \frac{f}{Z}-A_{c} \frac{f}{2 Z^{2}} L \theta
\end{aligned}
$$

We define the feature value difference $\alpha=\alpha_{2}-\alpha_{1}$ as the new feature value in the visual walking system. The equation has the following form:

$$
\begin{aligned}
\alpha_{2}-\alpha_{1} & =A_{c} \frac{f}{Z}-A_{c} \frac{f}{2 Z^{2}} L \theta-A_{c} \frac{f}{Z}-A_{c} \frac{f}{2 Z^{2}} L \theta \\
\alpha & =-A_{c} \frac{f}{Z^{2}} L \theta
\end{aligned}
$$

Differentiating the both sides of Eq.(10) yields the following equation.

$$
\dot{\alpha}=-A_{c} \frac{f}{Z^{2}} L \dot{\theta}=A_{c} \frac{f}{Z^{2}} L \dot{\theta}_{c}
$$

, where $\dot{\theta}=-\dot{\theta}_{c}$ by the principle of the perspective transformation. Eq.(11) similarly to eq.(2) yields the simplified sampled data model by discretization each walking step.

$$
\alpha(k+1)=\alpha(k)+A_{c} \frac{f L}{Z^{2}} u_{r}(k) \Delta T
$$

, where $u_{r}(k)$ is the contorol input $u_{r}(k)=\dot{\theta}_{c}$ (in Fig.4). The characteristic Eq.(11) is defined only in the visual walking. The controlling the feature value $\boldsymbol{\xi}$ and feature value difference $\alpha$ enable tracking of the rotation target.

\section{Decoupled rotation motion control}

1) Translation control: We separate rotation motion control from translation motion control because these input interfere each other, which cause the offset of the position and angle[7]. The translation motion is 2-DOF, so that the image Jacobian to control $\boldsymbol{J}_{2 d} \in R^{8 \times 2}$ is shown in Eq.(13).

$$
\boldsymbol{J}_{2 d}=\left[\begin{array}{cc}
-\frac{f}{Z} & \frac{x_{1}}{Z} \\
0 & \frac{y_{1}}{Z} \\
-\frac{f}{Z} & \frac{x_{2}}{Z} \\
0 & \frac{y_{2}}{Z} \\
-\frac{f}{Z} & \frac{x_{3}}{Z} \\
0 & \frac{y_{3}}{Z} \\
-\frac{f}{Z} & \frac{x_{4}}{Z} \\
0 & \frac{y_{4}}{Z}
\end{array}\right]
$$

The translation control input $\boldsymbol{u}_{x z}(k)=\left[u_{x}(k), u_{z}(l)\right]^{T} \in$ $R^{2}$ is based on the state feedback, the following control law is used,

$$
\boldsymbol{u}_{x z}(k)=-\boldsymbol{K} \boldsymbol{J}_{2 d}^{+} \overline{\boldsymbol{\xi}}=-\boldsymbol{K} \boldsymbol{J}_{2 d}^{+}\left(\boldsymbol{\xi}-\boldsymbol{\xi}_{d}\right)
$$

, where $\boldsymbol{K} \in R^{2 \times 2}$ is feedback gain, $\boldsymbol{J}_{2 d}^{+} \in R^{2 \times 8}$ psudoinverse matrix of the image Jacobian $\boldsymbol{J}_{2 d}$.

The feedback gain is diagonal matrix which was decided by the pole placement method.

2) Rotation control: We predicted that the system Eq.(12) converge by simple state feedback control, the following control law is used,

$$
u_{R}(k)=-K_{R} \alpha(k)=-K_{R}\left(\alpha_{2}(k)-\alpha_{1}(k)\right)
$$

, where $K_{R}$ is feedback gain in the rotation control. Eq.(15) has a reasonably effect that decouple the Z-axis motion with respect to body coordinate system from other DOF.

3) walking trajectory: The control input Eq.(14) and Eq.(15) are parameters to generate the walking trajectory in the visual waking. The translation control input is the length of the stride and the side stepping, the rotation control input is the angle of the turn.

\section{STABILIZATION}

We verify the stability of the proposed control law. New feature value vector $\boldsymbol{\omega} \in R^{9}$ is defined as $\boldsymbol{\omega}=[\boldsymbol{\xi}, \alpha]^{T}$. The 
define of the control input $\boldsymbol{u}_{\mathbf{3}}$ is same. New system equation is

$$
\boldsymbol{\omega}(\boldsymbol{k}+\mathbf{1})=\boldsymbol{\omega}(\boldsymbol{k})+\boldsymbol{J}_{s} \boldsymbol{u}_{3}(k) \Delta T
$$

, where

$$
\boldsymbol{J}_{s}=\left[\begin{array}{cc}
\boldsymbol{J}_{3 d} \\
\mathbf{0}_{2}^{T} & A_{c} \frac{f L}{Z^{2}}
\end{array}\right] \in R^{9 \times 3} .
$$

The control law is Eq.(18) from Eq.(14) and Eq.(15).

$$
\begin{aligned}
\boldsymbol{u}_{3}(k) & =\left[\begin{array}{c}
\boldsymbol{u}_{x z}(k) \\
u_{r}(k)
\end{array}\right]=-\left[\begin{array}{cc}
\boldsymbol{K}_{2 d}^{+} & \mathbf{0}_{2} \\
\mathbf{0}_{8}^{T} & K_{R}
\end{array}\right]\left[\begin{array}{l}
\boldsymbol{\xi} \\
\alpha
\end{array}\right] \\
& =-\left[\begin{array}{cc}
\boldsymbol{K} & \mathbf{0}_{2} \\
\mathbf{0}_{2}^{T} & K_{R}
\end{array}\right]\left[\begin{array}{cc}
\boldsymbol{J}_{2 d}^{+} & \mathbf{0}_{2} \\
\mathbf{0}_{8}^{T} & 1
\end{array}\right]\left[\begin{array}{c}
\boldsymbol{\xi} \\
\alpha
\end{array}\right] \\
& =-\boldsymbol{K}_{w} \boldsymbol{J}_{w} \omega(k)
\end{aligned}
$$

, where $\boldsymbol{K}_{w} \in R^{3 \times 3}, \boldsymbol{J}_{w} \in R^{3 \times 9}$. It is known that the system Eq.(16) is uncontrollable because the components of the feature value $\boldsymbol{\xi}$ are allowed that they move independent[8]. We apply the transformation $\boldsymbol{z}(k)=\boldsymbol{J}_{s}^{T} \boldsymbol{\omega}(k)$ to constrain the movement of $\boldsymbol{\xi}$, we get Eq.(19).

$$
\boldsymbol{z}(k+1)=\boldsymbol{z}(k)+\boldsymbol{J}_{s}^{T} \boldsymbol{J}_{s} \Delta T \boldsymbol{u}_{3}(k)
$$

The state feedback of Eq.(19) is

$$
\boldsymbol{u}_{3}(k)=-\overline{\boldsymbol{K}} \boldsymbol{z}(k)=-\overline{\boldsymbol{K}} \boldsymbol{J}_{s}^{T} \boldsymbol{\omega}(k) .
$$

The substituting Eq.(20) to Eq.(19) yield Eq.(21) to velify the stability.

$$
\boldsymbol{z}(k+1)=\left(\boldsymbol{I}-\boldsymbol{J}_{s}^{T} \boldsymbol{J}_{s} \overline{\boldsymbol{K}} \Delta T\right) \boldsymbol{z}(k)
$$

The stable condition of the digital control system is that the all poles of $\left(\boldsymbol{I}-\boldsymbol{J}_{s}^{T} \boldsymbol{J}_{s} \overline{\boldsymbol{K}} \Delta T\right)$ must be located within a unit circle. $\boldsymbol{K}_{w}$ is written $\boldsymbol{K}_{w}=-\overline{\boldsymbol{K}} \boldsymbol{J}_{s}^{T} \boldsymbol{J}_{w}^{-1}$ from Eq.(18) and Eq.(20). The eigenvalue $\lambda$ is $-0.12,0,0.8$ on condition that $\boldsymbol{K}=\operatorname{diag}(0.1,0.5), K_{R}=0.01$, the sampling time $\Delta T=$ $2.0 \mathrm{sec}$. The decoupled rotation motion control can stabilize the system.

\section{Simulation AND Experiment}

Using the feature value difference, we reached the control law to stabilize the visual walking. The stability is also sufficient. We verify the it by the simulation and the experiment.

\section{A. Simulation}

We simulate tracking the rotation target on condition that target rotate $\theta=\frac{\pi}{4}$ rad at time $t=0 \mathrm{sec}$. The result of the body posture is shown in Fig.6. The results of the feature value $\boldsymbol{\xi}$ are shown in Fig.7,8, 9 and 10. The result of amplitude difference is shown in Fig.12. We show the results of the generalized inverse matrix law in these figure for comparison.

In Fig.6, the angle of the robot converge to the reference angle $\left(\frac{\pi}{4} \mathrm{rad}\right)$ in the proposed law though the previous law cannot converge to the reference. From Fig.11 and 12, the rotation control converge faster than the translation control. This means that the rotation system hardly ever interfere with the translation system. Second, we verify the degree of the

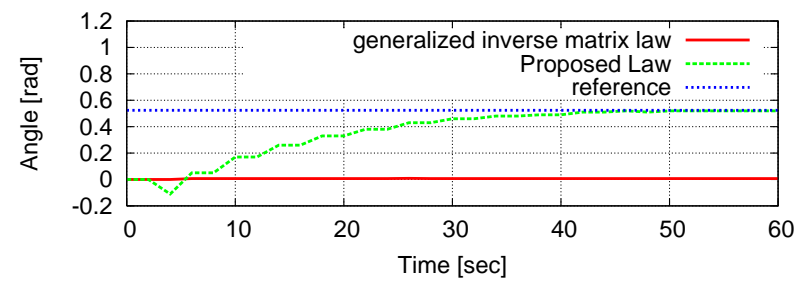

Fig. 6. Body posture with respect to world coordinate system(Simulation)

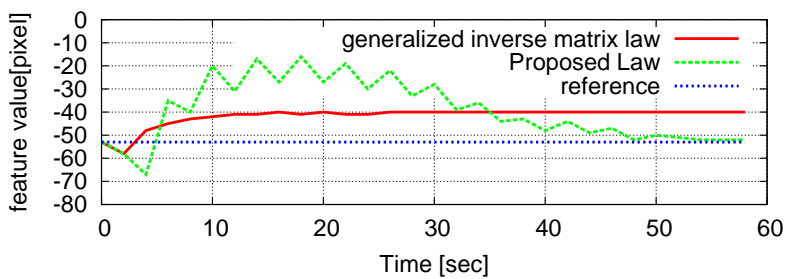

Fig. 7. Feature value $x_{1}$ in the feature point $\xi_{1}$ (Simulation)

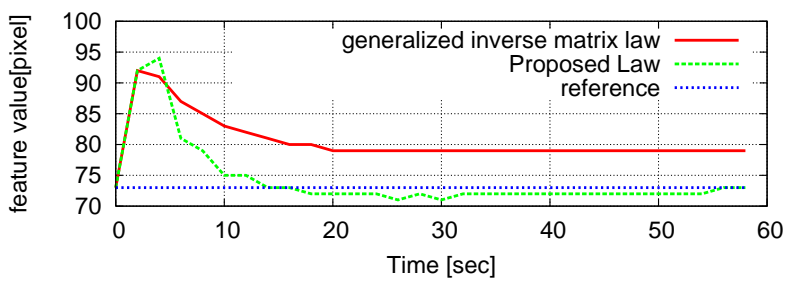

Fig. 8. Feature value $y_{1}$ in the feature point $\xi_{1}$ (Simulation)

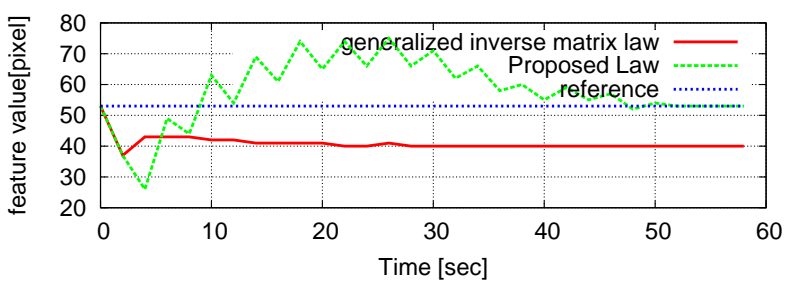

Fig. 9. Feature value $x_{2}$ in the feature point $\xi_{2}$ (Simulation)

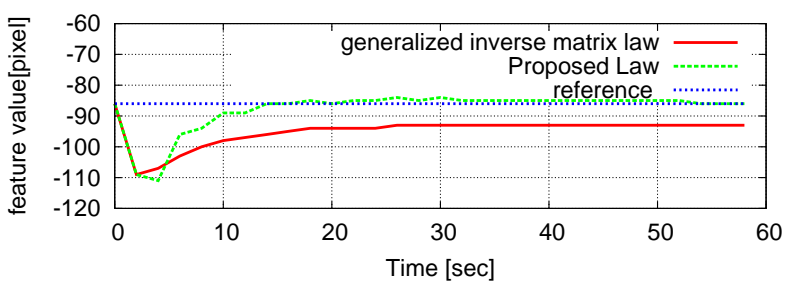

Fig. 10. Feature value $y_{3}$ in the feature point $\xi_{3}$ (Simulation)

interference. The pole of the system locate within a unit circle and the simulation is also stable on condition that the distance between target and robot is $Z>0.37 \mathrm{~m}$. The rotation control is hardly interfered in translation control within the limits. The proposed control law is valid for the practical applications.

\section{B. Experiment}

1) Hardware: The more on the biped robot MARI-2 developed by authors can be found in [1]. It has 12-DOF and the height and the weight are $1.3[\mathrm{~m}]$ and $70[\mathrm{~kg}]$. It has $12 \mathrm{AC}$ servo motors, six of which are for one leg. It has Force/Torque 


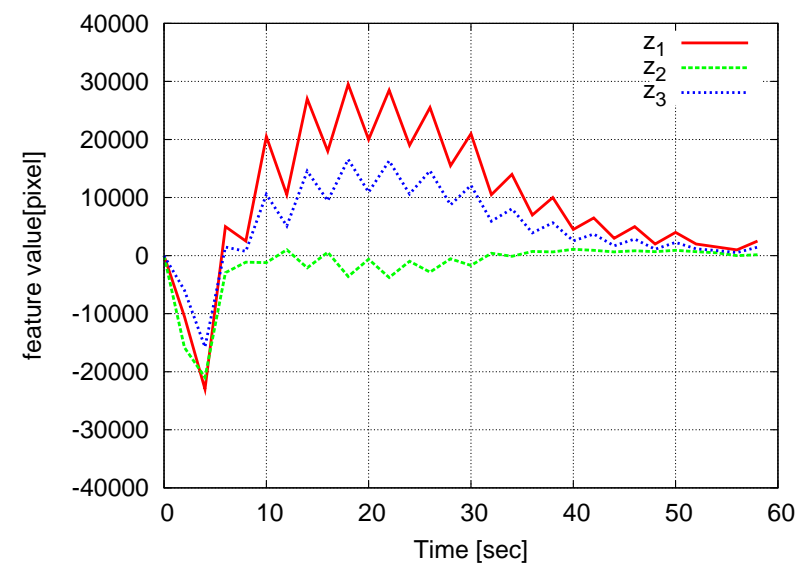

Fig. 11. Transformed Feature value $z(k)$ and theoretical response (Simulation)

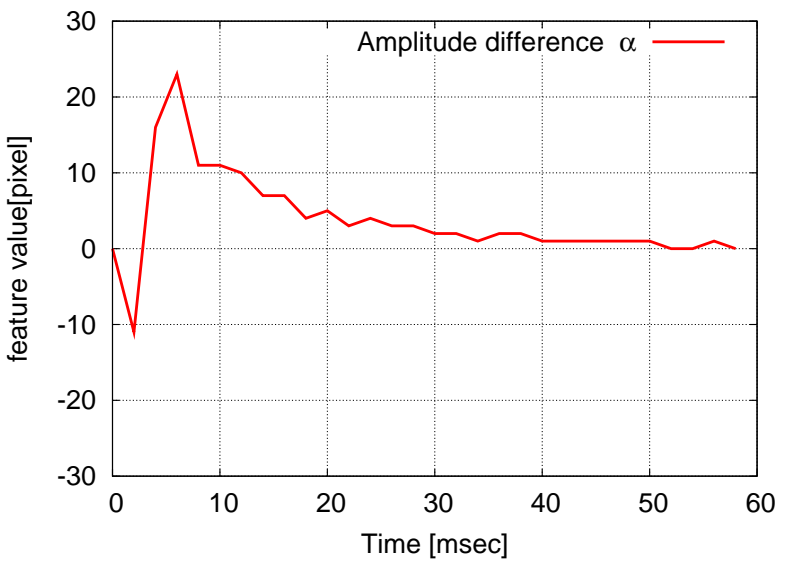

Fig. 12. Feature value amplitude difference $\alpha$ and theoretical response (Simulation)

sensors as the ZMP sensors under each sole and two CCD cameras on the head. The OS is based on the RT-Linux, and all of controllers and servo amplifiers are on the robot body, thus it is called autonomous walking robot.

2) Experimental result: The experimental results of the feature value by the proposed law and the previous law are shown in Fig.13-16 and Fig.17-20. The transformed feature values are shown in Fig.21. The amplitude difference is shown in Fig.22.

The offset of the feature value exist in Fig.17-20. The proposed control law reduce it(Fig.13-16). The response of the rotation system looks faster than the response of the translation system as the simulation result(Fig.21 and 22).

The responses are similar to the simulations though the speed of the convergence and the peak value of the response are different. In Fig.22, the feature value amplitude difference oscillates while the robot is walking compared with simulation, however the tendency of the response is similar. We expect that the reason of the oscillation is nonlinear factor that is the roughness of the machining accuracy and the asymmetric diversity of the robot by the deformation and the delay of the

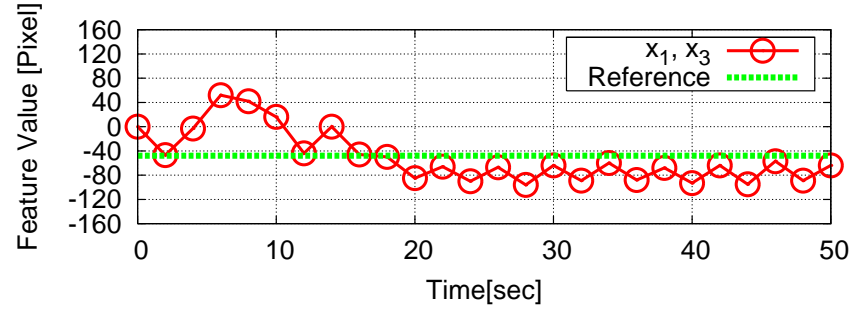

Fig. 13. Feature value $x_{1}$ in the feature point $\xi_{1}$ by Proposed law (Experiments)

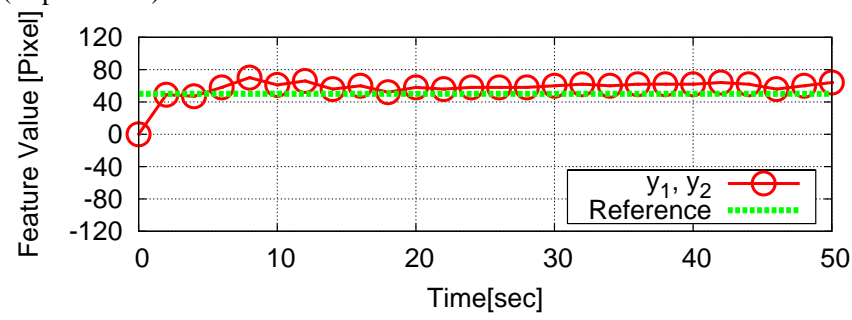

Fig. 14. Feature value $y_{1}$ in the feature point $\xi_{1}$ by Proposed law (Experiments)

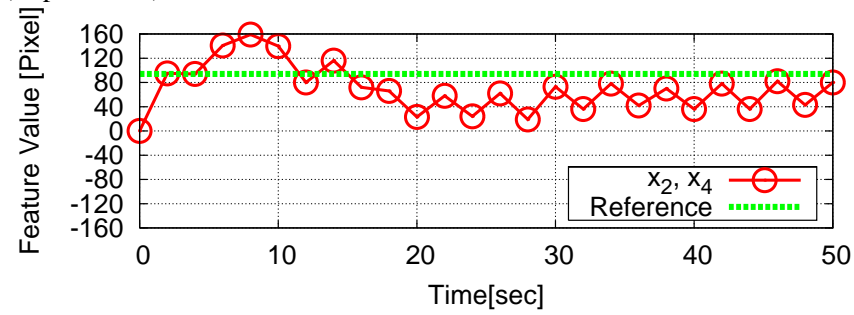

Fig. 15. Feature value $x_{2}$ in the feature point $\xi_{2}$ by Proposed law (Experiments)

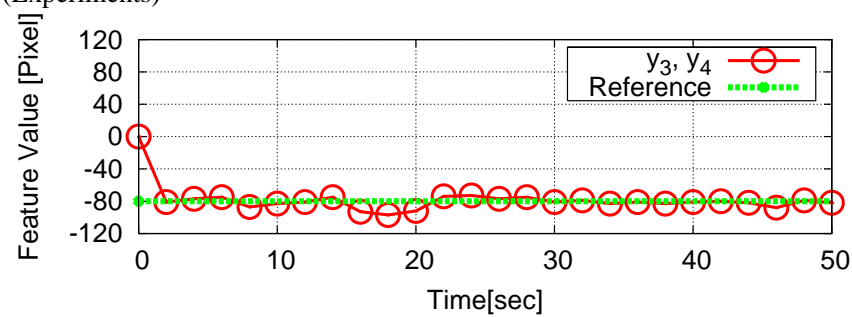

Fig. 16. Feature value $y_{3}$ in the feature point $\xi_{3}$ by Proposed law (Experiments)

image processing and so on.

The experimental measurement between robot and target object is shown Table I. The feature value and amplitude difference converge to reference, so that the body angle and Yaxis offset are smaller than previous control law with respect to the body coordinate system. The proposed control law is effective to follow the rotation object with the walking robot.

\section{Conclusion}

In this paper, we proposed a new control law to track the rotated target object using the characteristic of the walking, which considered the interference between translational motion and rotational motion. The decoupling is realized by simulations and experiments under the condition that the 


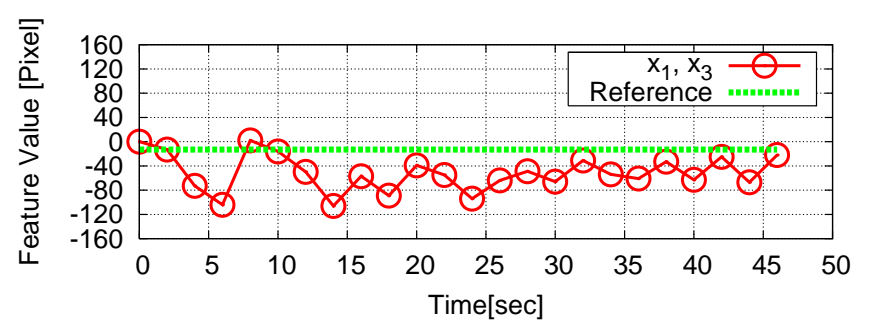

Fig. 17. Feature value $x_{1}$ in the feature point $\xi_{1}$ by previous law (Experiments)

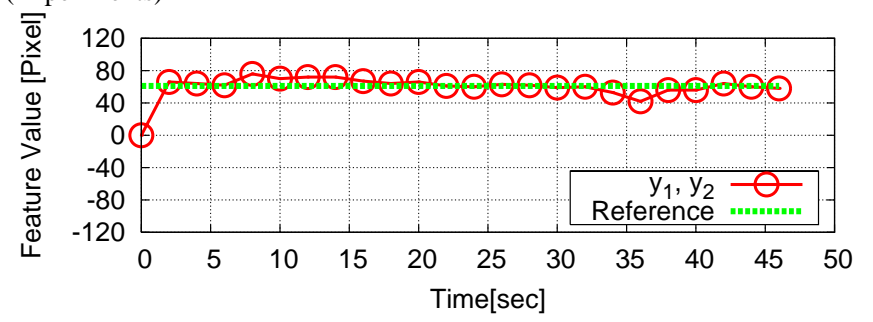

Fig. 18. Feature value $y_{1}$ in the feature point $\xi_{1}$ by previous law (Experiments)

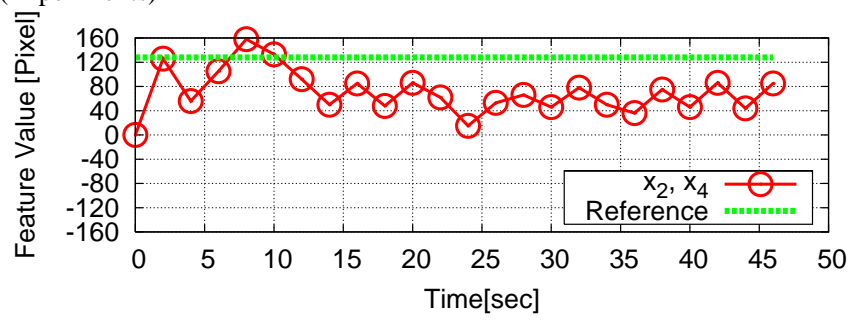

Fig. 19. Feature value $x_{2}$ in the feature point $\xi_{2}$ by previous law (Experiments)

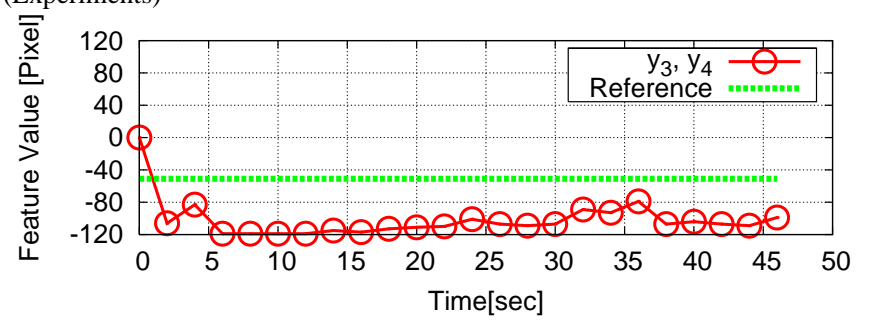

Fig. 20. Feature value $y_{3}$ in the feature point $\xi_{3}$ by previous law (Experiments)

distance between the target and the robot is larger than 0.37 $\mathrm{m}$. As a result, the walking robot tracked the translated and rotated target object without a practical issue.

\section{REFERENCES}

[1] K. Obiya, T. Takahashi, C. Zhu, and A. Kawamura, "Development of the biped walking robot mari-2," in Proc. of the 19th Annual Conference of the RSJ, September 2001, pp. 675-676.

[2] Y. Asano, C. Zhu, and A. Kawamura, "Visual walking for biped walking robot mari-2," in Proc. of the 8th IEEE International Workshop on Advanced Motion Control, Kawasaki, Japan, March 2004, pp. 399-402.

[3] K. Itoh and S. Sakane, "Visual tracking on dynamic transition in groups of affine templates," Robotics Society of Japan Trans, vol. 19, no. 1, pp. 100-108, 2001.

[4] K. Hashimoto, "Prediction and sensitivity of visual servo," SICE Trans, vol. 40, no. 9, pp. 630-635, 2001

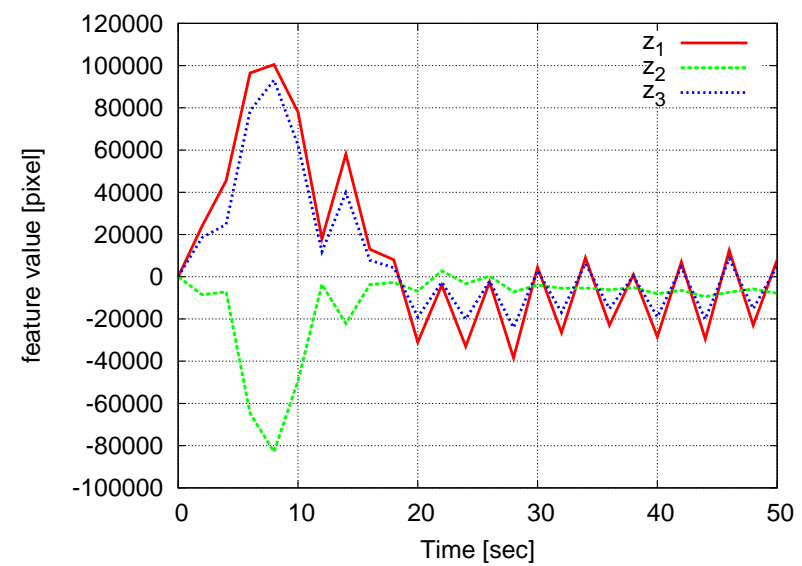

Fig. 21. Transformed Feature value $z(k)$ (Experiments)

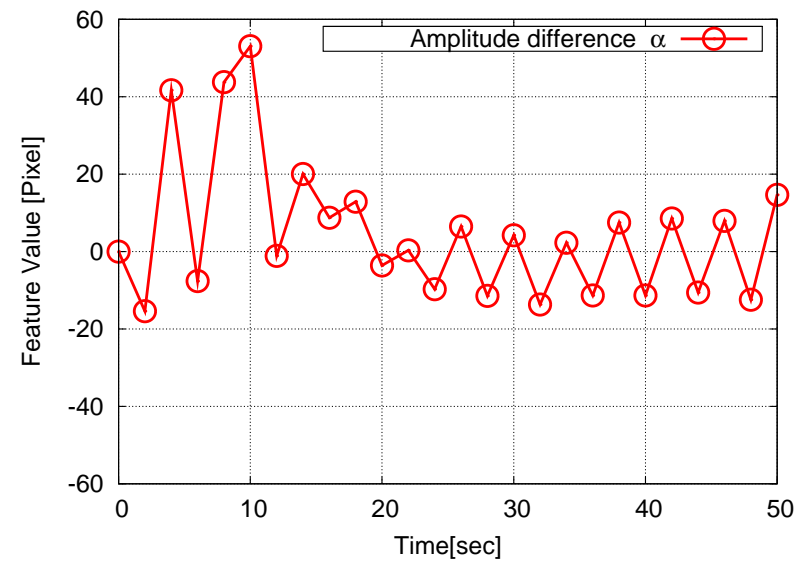

Fig. 22. Feature value amplitude difference $\alpha$ (Experiments)

TABLE I

EXPERIMENTAL MEASUREMENT RESULT

\begin{tabular}{ccc}
\hline & Proposed law & Previous law \\
\hline Body angle offset[rad] & 0.0 & $\frac{\pi}{9}$ \\
Y-axis offset $[\mathrm{m}]$ & 0.05 & 0.3 \\
\hline
\end{tabular}

[5] K. Hasimoto, T. Kimoto, T. Ebine, and H. Kimura, "Manipulator control with image-based visual servo," in Proceedings of IEEE ICRA, 1991, pp. 2267-2272.

[6] P. I. Corke and S. A. Hutchinson, "A new hybrid image-based visual servo control scheme," in Proceedings of the 39th IEEE Conference on Decision and Control, Sydney, Australia, Dec 2000, pp. 2521-2526.

[7] Y. Asano and A. Kawamura, "Stability on orientation motion of biped walking robot aiming at a target object," in Proc. of the 9th IEEE International Workshop on Advanced Motion Control, Istanbul, Turkey, March 2006, pp. 428-432.

[8] K. Hashimoto, T. Ebine, and H. Kimura, "Visual servoing -optimal control approach-," RSJ Trans., vol. 12, no. 5, pp. 773-778, July 1994. 